vollkommen anzuschliefsen, es würde somit unter 340 nur 우 von Asmara (No. 1010) fallen, und eine $340 \mathrm{a}$ mit den $\sigma^{\top} \sigma^{x}$ (No. 403, 430) als „Saxicola oenanthe rostrata Hempr. u. Ehrbg." einzufügen sein.

p. 87. Phoenicurus phoenicurus mesoleuca. Zur Systematik ist nachzutragen: In O. M. 1910. XII. p. 189 falst Sarudny die mesoleuca-Formen als eine besondere Gruppe auf, welche sich durch gröfsere Stumpfflüglichkeit von den phoenicurusVertretern unterscheide. Bei dieser Gelegenheit beschreibt er nun die beiden asiatischen Formen $P . m$. bucharensis und P.m. incognita. Bei meinem Stück ist die 2. Schwinge um ein Vinimum läng e r als die 6, keineswegs aber kürzer, somit kann es sich hier nicht um bucharensis handeln. Da ferner auf dem Rücken keine schwarze Färbung vorhanden ist, dürfte mein $\sigma^{T}$ auch nicht zur Form incognita gehören, denn als mindestens semiad. mufs ich es ansprechen.

p. 90. Bei Art 363 mufs es statt ,Melittophagus fusillus cyanostictus" heifsen ,M. pusillus c."

p. 92. Art 345 mufs statt "Saxicola pleschanka Lepech" heifsen "Saxicola pleschanka pleschanka Lepech".

\title{
Dr. Freiherr Richard König von und zu Warthausen.
}

Ein Gedenkblatt.

(Mit Bildnis.)

Mit besonderer Freude wird der Blick des Reisenden, der sich auf den rollenden Rädern der Bahn im Fluge von der alten Reichsstadt Ulm an den Bodensee tragen läfst, auf dem stattlichen Schlosse weilen, das sich unweit Biberach auf langgestrecktem Hügel stolz erhebt: eine Erquickung für das Auge nach der Einförmigkeit der oberschwäbischen Ebene, die doch so voller intimer Reize ist. Ehe wir aber an das schwäbische Meer weitereilen, wollen wir im Geiste hier rasten, wollen den Schlofsberg hinansteigen und im Parke des Schlosses Warthausen an einsamer Stätte des Schlofsherrn gedenken, der hier am 17. Januar 1911 die letzte Ruhe fand. Ein langes, innerlich reiches Leben lag hinter Freiherrn Richard König von und zu Warthausen, als er fast einundachtzigjährig am 14. Januar die Augen für immer schlofs. Ihm ward das Glück zuteil, auf eigener Scholle sein Leben zu verbringen, ,im echten Land, im Heimatland, auf eigner Weid' und Wonne", wie Kurwenal singt.

Mancherlei Erinnerungen verbinden sich mit Schlofs Warthausen. Hier weilte seit 1762 der ehemalige kurmainzische Staatsminister Graf von Stadion mit seinem Günstling, dem Hofrat La Roche, dessen Gattin die erste Geliebte des Dichters Christoph Martin Wieland war. Hier auf Warthausen wehte, nach Goethes Worten, den gewandten und anmutigen Dichter des „Oberon“ „in 
diesem angesehenen, wohl eingerichteten Hause zuerst die Weltund Hofluft an". Im Jahre 1827 kam das Gut Warthausen an den württembergischen Staat, 1829 an die Freiherrn von König, einer reichsadeligen, einst aus Niedersachsen und Elsals eingewanderten Familie, von welcher der im Besitz des Majorats Warthausen befindliche Zweig seit 1867 den Namen Freiherr König von und $\mathrm{zu}$ Warthausen führt.

Am 6. Februar 1830 wurde zu Warthausen Freiherr Richard geboren. Früh schon mag der aufgeweckte und begabte Knabe zu der Eigenart der ihn umgebenden oberschwäbischen Heimat sich hingezogen gefühlt haben, denn ihr gehörte seine Liebe sein ganzes Leben hindurch. Nachdem er in den Jahren 1842 bis 1846 in Ulm sich auf dem Gymnasium aufgehalten hatte, besuchte er nach Erstehung der Maturitätsprüfung die Universität Tübingen, die Forstakademie Tharand und die landwirtschaftliche Akademie Hohenheim bei Stuttgart, um an diesen Orten Forst- und Landwirtschaft $\mathrm{zu}$ studieren. Mit ganzer Seele aber warf er sich auf die ihm liebste Wissenschaft, auf die Ornithologie, in der er in der Folge zum Meister werden sollte. Es ist zwar richtig, dafs Baron König nicht besonders viel, insbesondere keine grölseren ornithologischen Werke veröffentlicht hat. Es hat fast den Anschein, als ob ihm das Schreiben, das schriftliche Fixieren seiner ausgedehnten und ausgezeichneten Beobachtungen, nicht besonders angenehm war. Aber das, was er einmal schrieb, hatte Hand und Fufs. Sein vorzügliches Gedächtnis setzte ihn in Stand, nach Jahren sich noch besonders beachtenswerter Beobachtungen aufs genaueste zu erinnern und diese, wenn er sich einmal an den Schreibtisch setzte, klar und anschaulich zum Ausdruck zu bringen. So knüpft er in der im Jahre 1886 veröffentlichten Abhandlung über die „Bauchschwangerschafte bei den Vögeln" an ein Vorkommnis aus dem Jahre 1851 an, in dem er in Tharand studierte und woselbst sich eines Tages das Gerücht verbreitete, die verwitwete Pastorin Täubert habe im Leib eines Huhnes ein ausgebildetes Küchlein gefunden. Er erzählt nun in humorvoller Weise, wie er die alte Dame, die sich an dem seltenen Funde „wie an einem Geschwür" geekelt habe, "zu Protokoll vernommen" habe und wie er dann diesen Fall in der Naumannia von 1854 (S. 34) zur öffentlichen Kenntnis gebracht und in jugendlichem Sinne sich einiges Aufsehen davon versprochen habe. Allein er sei übel weggekommen: Die damaligen mafsgebenden Ornithologen, Dr. L. Thienemann in Dresden, sein alter Lehrer und Freund, Dr. Gloger und Dr. Julius Hoffmann äufserten sich abfällig und ablehnend über die Entdeckung des jungen Ornithologen, der - tatsächlich doch recht hatte.

Das glühende Interesse, das er für die Ornithologie hatte, brachte ihn auch in persönliche Verbindung mit den damals lebenden grofsen Ornithologen, mit denen ihn zum Teil herzliche Freundschaft verband. So kannte er Naumann, die beiden Brehm, 
den schon genannten Thienemann, J. H. Blasius, Eugen von Homeyer, Reichenbach, Cabanis, Heuglin, die ihm alle schon vorangegangen sind und mit denen er zum Teil auch in brieflichem Verkehr stand. Vielleicht gelingt es noch, diesen Briefwechsel wenigstens teilweise zur Veröffentlichung zu bringen.

Nach Abschlufs seiner Studien widmete er sich der Verwaltung des Gutes Warthausen, dabei eifrig auf allen Gebieten der Naturwissenschaften weiter studierend, forschend und sammelnd. In der Ornithologie hatte es es ihm besonders die Oologie und damit die Fortpflanzungsgeschichte der Vögel angetan. In dieses Gebiet fallen auch die meisten seiner Publikationen. So schrieb er schon im Jahre 1859 über „Zur Fortpflanzungsgeschichte der Spottsänger“, 1860 ,Zur Fortpflanzungsgeschichte des Europäischen Seidenschwanzes", 1853 über die Fortpflanzung der Kiebitzartigen Vögel $u$. a. m. Hand in Hand mit diesen litterarischen Arbeiten ging seine eifrige Sammeltätigkeit, die sich hauptsächlich auf Eier und Nester erstreckte. Es ist bekannt, dafs Baron König eine der schönsten und wertvollsten privaten Eiersammlungen zusammengebracht hat. Einer brieflichen Nachricht seines ältesten Sohnes zufolge, des derzeitigen Majoratsherrn, Amtsrichters Freiherrn Hans von König-Warthausen, dem ich mancherlei für diesen Bericht verdanke, hat insbesondere der württembergische Afrikareisende Theodor von Heuglin, der mit Richard von König-Warthausen eng befreundet war, in Afrika, speziell im Sudan, aber auch im Norden Eier für die Warthausen'sche Sammlung, in der insbesondere die Eier der amerikanischen Waldhühner, der Raubvögel, Sumpf- und Wasservögel gut vertreten sind, zusammengebracht. Diese Sammlung bleibt im Besitz der Familie. Sie ist dem Bruder des Majoratsherrn, Freiherrn Fritz von König-Warthausen zugewiesen, von dem wir aus den Schriften des alten Herrn erfahren, dafs er, wie auch seine Schwester Elisabeth, jetzt Frau von Alberti, ein eifriger und kenntnisreicher Ornithologe ist. Bei der grofsen Liebe, die unseren Forscher zur Tierwelt beseelte, ist es nicht zu verwundern, dafs er diese Eigenschaft auch auf seine Kinder übertrug, die ihn in der Wartung der verschiedensten Tiere, die er pflegte, unterstützten. Insbesondere habe sich Baron König, so berichtet sein ältester Sohn, Eulen, Meisen, rabenartige Vögel, Rebhühner und früher auch Wiedehöpfe gehalten.

Als Beispiel, wie innig Freiherr von König mit den von ihm gepflegten Tieren verkehrte, mag eine Schilderung aus seiner eigenen Feder dienen. Er schreibt in dem „Naturwissenschaftlichen Jahresbericht von 1891" in den Jahresheften des Vereins für vaterländische Naturkunde in Württemberg, Jahrgang 1894 bei der „Waldohreule“ folgendes:

„Am 1. Juli (1891), also aufserordentlich verspätet, wurde mir eine Waldohreule noch im Dunenkleid überbracht, welche Kinder beim Beerensuchen im Fichtenhochwald am Boden 
gefunden hatten. Ich zog den Vogel auf und machte ihn zu meinem Schlafzimmergenossen. Sobald er flugbar war, wurde er Nachts in einem kleinen Käfig bewahrt, den ihm der Diener in der ersten Morgenfrühe zu öffnen pflegte. Dauerte die Haft zu lange oder war er besonders heiter, so liefs er seine Stimme, meist gedämpft, hören. Zuerst setzte er sich dann auf die Brüstung eines mein Bett umgebenden Verschlags, wo er eine Menge Verbeugungen gegen mich und die komischsten Capriolen machte; von hier ging er auf meine weiche Bettdecke, wo unter Wirkung dieses elastischen Podiums die sonderbarsten Grotesktänze regelmäfsig aufgeführt wurden, bis er sein bereitgestelltes Frühstück aus der Hand empfing; nachher safs er oft noch stundenlang auf dem Kopfstück meiner Bettlade, sich ab und zu herabbeugend, um meinem Atem zu lauschen oder mir die Haare zu krauen; wurde ihm meine Morgenruhe zu lang, so begab er sich auf den Waschtisch, wo er im halbgefüllten Waschbecken „Wasser trat", auch einen Badeschwamm ganz klein zerbissen hat, von dem er behufs der „Gewöllbildung“ Teile verschluckte. Sein gröfstes Interesse erregte stets das Plätschern von Wasser; wenn ich solches eingofs, mich wusch u.s.w.; trat er stets beobachtend nächst zur Stelle, womöglich um sich auf den Rand der Gefässe zu setzen. Manchmal hat er mich auch damit überrascht, dafs er übrige Mäuse unter meinen Effekten aufbewahrte. Immanuels (er hörte auf den Ruf „Immo“) Aufenthalt den Tag über war auf der Höhe eines geöffneten Kleiderschranks oder der Zimmertüre oder auf der Lehne eines Stuhls, die bei Sonnenschein mit einem aufgespannten Regenschirm überschattet wurde. Da an diesen fixen Plätzen stets Papierbögen unterlegt waren, kamen Verunreinigungen des Zimmers kaum vor. Über Tags trug ich öfter den Vogel auf der Schulter durch's Haus oder in den Garten, nur Abends machte er leise Flüge im Zimmer. Bei einem solchen geriet er hinter einen schweren Kasten und wurde bei seiner Befreiung schwer verletzt. In einem Kistchen liegend wurde er noch mehrere Tage gewaltsam gefüttert, am Morgen des 29. September starb er in meiner Hand mit einem Blick, den nur der Tierfreund versteht, und unter einem furchtbaren Aufschrei. Dieser Unfall hat mich so tief ergriffen wie einst der jähe Tod eines Wachtelkönigs, der auf dem Schreibtisch den Zügen meiner Feder folgte und auf meinem Kopfkissen zu übernachten pflegte. Wie sehr die Tierseele, wenn richtig behandelt, dem Menschen sich anschliefst, begreifen Oberflächlichkeit und Spott freilich nicht."

Die meisten der Veröffentlichungen aus der Feder Baron Königs sind in den oben erwähnten Jahresheften des Vereins für vaterländische Naturkunde in Württemberg erschienen, deren letzter Jahrgang (1911) einen warmherzigen Nekrolog über unseren Forscher von Oberstudienrat Dr. K. Lampert bringt. Schon im Jahre 1853 trat er diesem Vereine als Mitglied bei, das Jahr 
1898 brachte ihm die Ehrenmitgliedschaft. In dem Vereinsorgan veröffentlichte Baron König von 1885 an seine „Naturwissenscbaftlichen Jahresberichte" aus Württemberg, zu welchen ihm eine Reihe naturwissenschaftlich und ornithologisch durchgebildeter Männer Beiträge lieferten, die er dann verarbeitete. Neun solcher Jahresberichte, in denen der Eigenart ihres Bearbeiters entsprechend die Ornithologie die führende Stelle hat, hat von König veröffentlicht; dann aber hörten sie bedauerlicher Weise auf, da die Beiträge immer spärlicher eingelaufen z. T. auch ganz ausgeblieben waren. Eine eifrige Tätigkeit entfaltete Baron König als Vorstand des „Molasseclubs“, eines Vereins oberschwäbischer Naturwissenschaftler, der sich im Jahre 1874 dem Verein für vaterländische Naturkunde angliederte. Zunehmende Altersbeschwerden, insbesondere ein langjähriges Gichtleiden, liefsen ihn vom Vereinsleben im Jahre 1898 zurücktreten. Im Jahre 1904 war in den „Jahresfesten" seine letzte Abhandlung "Nordische Wintergäste", eine Arbeit über den Seidenschwanz, erschienen. Der Tag hatte sich geneigt.

Mancherlei Ehren waren in seinem langen Leben ihm zuteil geworden: er war königlich württembergischer Kammerherr, Rechtsritter des Johanniterordens, als welcher er im Feldzuge 1870/71 Verpflegungszüge nach Frankreich führte und wofür ihm das Eiserne Kreuz am weifsen Bande verliehen wurde, ordentliches und korrespondierendes Mitglied einer Reihe von naturforschenden Gesellschaften, ritterschaftlicher Abgeordneter der württembergischen Ständekammer, der er von 1862 bis 1894 angehörte und in der er als Autorität in allen Fragen des Vogelschutzes galt. $\mathrm{Zu}$ dem vom 17.-20. Mai 1891 in Budapest abgehaltenen zweiten internationalen ornithologischen Kongrefs wurde er als Delegierter der K. württ. Regierung entsandt. Auf jenem Kongrefs stand $u$. a. auch die Vogelschutzfrage zur Diskussion. Baron König ergriff bei der Debatte das Wort und führte aus - wofür er schon lange durch Wort und Schrift und Vorbild eingetreten war, als die Heimatschutzbestrebungen noch nicht so aktuell waren wie jetzt -, seine persönliche Auffassung gehe dahin, er sei nicht in der Lage, einen scharfen Unterschied zwischen nützlichen und schädlichen Vögeln machen zu können, da diese Begriffe sehr relativer Art seien und nach Berufsarten und nach Örtlichkeit sich oft recht verschieden auslegen lassen. Jedes Geschöpf habe seine eben durch die Existenz bewiesene Existenzberechtigung; allerdings sei der Mensch, und zwar gleichfalls auf Grund seiner Daseinsberechtigung, befugt, sich der Nebengeschöpfe zu erwehren oder sie sich nutzbar zu machen. Er habe aber als Gegenleistung die moralische Pflicht, sie da zu schützen, wo sie indifferent sind, ihm nicht unbedingt oder erheblich schaden, oder wo sie evident nützen. Die völlige Ausrottung selbst einer enschieden schädlichen Art erscheine vom naturwissenschaftlichen Standpunkt, welcher die „Erhaltung der 
Art" zu begünstigen hat, verwerflich. (Jahreshefte des Vereins für vaterl. Naturkunde 1892 S. 58.) -

Überblickt man das Lebenswerk des Freiherrn von König, so empfindet man es als einen Akt der Gerechtigkeit und begrüfst es, dafs die naturwissenschaftliche Fakultät der Universität Tübingen ihm den naturwissenschaftlichen Doktor honoris causa verlieh. Mit Genugtuung mag der also Gefeierte diese Ehrung entgegengenommen haben. - Uns aber wird es wehmütig ums Herz in dem Gedanken, dafs wieder einer der alten Garde, einer der Naumann und die beiden Brehm und all die hochverdienten Forscher der Mitte des vorigen Jahrhunderts noch kannte, von uns geschieden ist, und keiner der geringsten! Trauernd stehen wir an der letzten Ruhestätte des Heimgegangenen; aber nicht ohne Erhebung gehen wir von dannen, daran denkend, dafs das, was der Entschlafene gelebt, geschrieben und gesprochen, nicht umsonst war. Ein treuer Sohn seiner Heimat, ein echter Naturforscher auf hoher Warte stehend war der Freiherr König von und zu Warthausen!

Es möge nun noch ein Verzeichnis der Schriften des Freiherrn von König folgen. Der schon erwähnte Sohn des Verstorbenen, der jetzige Majoratsherr, hatte die Freundlichkeit, da ich selbst nur einen Teil der Arbeiten des Barons König besafs, alles, was sich an Schriften seines Vaters vorgefunden hat, mir zuzusenden. Es war das ein Paket von märsigem Umfang, das die Aufschrift von der Hand des alten Herrn trug „Eigene Arbeiten". Es ist sehr wahrscheinlich, dafs nicht alle Arbeiten des Freiherrn von König in diesem Paket vereinigt waren und so konnte, da die Zeit drängte, eine lückenlose Aufzählung der aus der Feder des Entschlafenen herrührenden wissenschaftlichen Arbeiten nicht geboten werden. Die meisten Sachen sind in dem Organ des Vereins für vaterländische Naturkunde in Württemberg (Stuttgart, Kgl. Hofbuchdruckerei Zu Gutenberg [Klett und Hartmann]) - im Nachfolgenden nur mit „Jahreshefte“ zitiert - enthalten.

a. Schriften nicht ornithologischen Inhalts:

1. Eine achtfach blühende Agave americana (Erscheinungsort?).

2. Beitrag zur Fauna Württembergs (über die Zwergmaus, Mus minutus Pall. und über die rote sibirische Feldmaus, Hypudaeus rutilus Illig. und Pall. u. a.), Jahreshefte 1855, S. 1.

3. Verzeichnis der Wirbeltiere Oberschwabens I. Abteilung: Säugetiere, veröffentlicht 1875 in den „Mitteilungen vom oberschwäbischen Zweigverein für vaterländische Naturkunde".

4. Nekrolog des Freiherrn Carl Franz August Sebastian von Schertel. (Erscheinungsort?)

5. Zur Erinnerung an Theodor von Heuglin, Journal für Ornithologie Januar-Heft 1877. 
Dr. Freiherr Richard König von und zu Warthausen.

6. Aus der Tierwelt (Beobachtungen über den Siebenschläfer oder die gemeine Haselmaus (Myoxus glis Schreb.), über das Hegen von Rehen und Wildschweinen u. a.), Jahreshefte 1885 S. 68.

\section{b. Schriften ornithologischen Inhalts:}

1. Leucopathie und andere Abänderungen der Normalfärbung bei Vögeln. (Erscheinungsort und -Jahr?)

2. Zur Fortpflanzungsgeschichte der Spottsänger, Moskau, Buchdruckerei der Kaiserlichen Universität 1859.

3. ZurFortpflanzungsgeschichte desEuropäischenSeidenschwanzes (Ampelis Linn., Bombycilla garrula Briss.), Moskau, Buchdruckerei der Kaiserlichen Universität 1860.

4. Über die Fortpflanzung der geierartigen Raubvögel, Buch der Welt 1860 S. 304.

5. Über die Fortpflanzung der Kiebitzartigen Vögel, Buch der Welt 1863 S. 279.

6. Bemerkungen über die Fortpflanzung einiger Caprimulgiden, Journal für Ornithologie 1868 S. 361.

7. Zur älteren Literatur (über Alca [Plautus] impennis, Salanganen-Nester, Zurückweisung des seinerzeit Dr. L. Thienemann ungerechterweise gemachten Vorwurfs, dieser habe einst das in seiner Sammlung befindliche Ei des Brillen-Alks, gelockt von der Versuchung so seltenen Besitzes, widerrechtlich zurückbehalten), Journal für Ornithologie 1869 S. 246.

8. Über die zur Unterscheidung der Vogeleier dienenden Merkmale, Jahreshefte 1876 S. 1.

9. Über das Verhalten verschiedener Nistvögel gegenüber dem Menschen, Jahreshefte 1884 S. 306.

10. Über die Gestalt der Vogeleier und über deren Monstrositäten, Jahreshefte 1885 S. 289.

11. „Bauchschwangerschaft" bei Vögeln, Jahreshefte 1886 S. 316.

12. Die Kreuzschnäbel und ihre Fortpflanzung. Eine monographische Studie, Jahreshefte 1889 S. 1.

13. Bericht über den 17.-20. Mai 1891 zu Budapest abgehaltenen zweiten internationalen ornithologischen Congrefs, Jahreshefte 1892 S. 32.

14. Ornithologischer Jahresbericht 1885, Jahreshefte 1876 S. 146. 15-22. Naturwissenschaftliche Jahresberichte ${ }^{1}$ ) aus den Jahren 1886-1893, veröffentlicht in den Jahresheften 1887-1896.

23. Über den Nestbau der Vögel, Jahreshefte 1890 S. 241.

24. Nordische Wintergäste (über den gemeinen Seidenschwanz, Bombycilla garrula Vieill.), Jahreshefte 1904 S. 287.

Heilbronn a. Neckar.

Staatsanwalt Walther Bacmeister.

1) hauptsăchlich ornithologischen Inhalts. 


\section{$2 \mathrm{BHL}$ Biodiversity Heritage Library}

1911. "Dr. Freiherr Richard König von und zu Warthausen." Journal fu

r Ornithologie 59, 613-619. https://doi.org/10.1007/bf02089571.

View This Item Online: $\underline{\text { https://www.biodiversitylibrary.org/item/43503 }}$

DOI: https://doi.org/10.1007/bf02089571

Permalink: https://www.biodiversitylibrary.org/partpdf/142774

\section{Holding Institution}

MBLWHOI Library

\section{Sponsored by}

MBLWHOI Library

\section{Copyright \& Reuse}

Copyright Status: No known copyright restrictions as determined by scanning institution.

This document was created from content at the Biodiversity Heritage Library, the world's largest open access digital library for biodiversity literature and archives. Visit BHL at https://www.biodiversitylibrary.org. 